\title{
Preparation of cross-sectional transmission electron microscopy specimens of obliquely deposited magnetic thin films on a flexible tape
}

\author{
Enrico G. Keim ${ }^{\text {a) }}$ \\ MESA + Research Institute, Central Materials Analysis Laboratory, University of Twente, \\ P.O. Box 217, 7500 AE Enschede, The Netherlands \\ Martin D. Bijker ${ }^{\text {b) }}$ and J. Cock Lodder \\ MESA + Research Institute, Information Storage and Technology Group (ISTG), University of Twente, \\ P.O. Box 217, 7500 AE Enschede, The Netherlands
}

(Received 10 August 2000; accepted 9 October 2000)

\begin{abstract}
In this article a method is described for preparing cross sections of obliquely deposited metal thin films on polymer substrates for transmission electron microscopy (TEM) observation. The layers of interest are brittle in nature and therefore it is difficult to obtain suitable TEM samples with conventional methods. Apart from the sample preparation method some details of the deposition technique will be elucidated. (C) 2001 American Vacuum Society. [DOI: 10.1116/1.1330259]
\end{abstract}

\section{INTRODUCTION}

Specimen preparation is an essential part of transmission electron microscopy (TEM). TEM specimens must be electron transparent and representative of the material one wants to study. In most cases it is desirable to have specimens which are uniformly thin, stable under the electron beam, conducting, and nonmagnetic. Clear exceptions are of course magnetic materials; besides structural information the investigation of magnetic structures may also be needed, e.g., with Lorentz microscopy. For metals or alloys in which crystal defects (dislocations) have to be studied, the specimens should not be made too thin to avoid possible annihilation of these defects.

There are many ways to prepare specimens for TEM. The chosen method will depend on both the type of material and the information one needs to obtain. The most important aspect to bear in mind is that the preparation technique must never affect what one observes or measures. In general, if one is interested in interface and/or film properties one should prepare a TEM specimen in cross section.

Upon surveying the literature, no recipe is provided for the preparation of TEM cross sections of metal sputtered (MS) or metal evaporated (ME) magnetic thin films on polymer substrates. Various private communications in the field $^{1,2}$ reinforce our impression that no adequate preparation procedure for this class of materials is available at present. However, proper quality TEM images of ME tapes in cross section have been published previously by SONY Corp. ${ }^{3-6}$ and by ourself ${ }^{7,8}$ without an adequate description of the TEM specimen preparation. Also, the company $\mathrm{BASF}^{9}$ has published some results, however, again without mentioning the preparational details of the TEM specimen. Most probably a microtome was used to prepare the TEM samples in the above-mentioned literature.

The use of a microtome has the drawback of cutting a

\footnotetext{
${ }^{a)}$ Author to whom correspondence should be addressed.

${ }^{b)}$ Present address: Onstream B.V., Lodewijkstraat 1, 5652 AC Eindhoven, The Netherlands.
}

combination of a ductile, fragile metal layer on top of a tough polymer. The latter is typically of $10 \mu \mathrm{m}$ thickness, while the layer of interest is in the $200 \mathrm{~nm}$ range. Moreover, the polymer may easily loose its mechanical stiffness when made thin. Therefore, a brittle TEM sample remains that can withstand only small mechanical forces during the final stages of sample handling.

For planar sample preparation of these types of layers several methods have been proposed. Dissolving the polyester substrate in a 1:3 mixture of trifluoroacetic acid and dichloromethane (see Ref. 10), or a 1:1 mixture of hexapropanol to chloroform $\left(\mathrm{CHCl}_{3}\right)$ might be considered. ${ }^{11}$ Furthermore, immersion in hydrazine monohydrate ${ }^{12}$ was succesfully employed. However, we did not attempt this method, since the focus of this article is on TEM samples for cross-section views.

Before describing the details of the cross-sectional TEM preparation method the oblique-deposition technology will be briefly discussed.

\section{PREPARATIONAL DETAILS OF THE DEPOSITION}

Sputtered tapes are prepared by similar means as metal evaporated tape (see, e.g., Ref. 13 and the formerly quoted Japanese publications ${ }^{3-6}$ ). Since they are still prepared in an experimental process, some details are given as to the preparational details. In Fig. 1 a schematic representation is shown of the so called miniroll coater. The films are sputtered at an oblique angle by two dc magnetron sputter guns situated at the bottom right-hand side of Fig. 1. Previous experiments have shown that the coercivity of obliquely deposited media can be enhanced during sputtering. ${ }^{13}$ This effect can be achieved even without adding oxygen to the process. By using two dc magnetron sputter guns it is possible to sputter either an underlayer or protective seed layer. The sputter guns were equipped with a flexmount, in order to change the angle between the substrate and the target. In addition to 


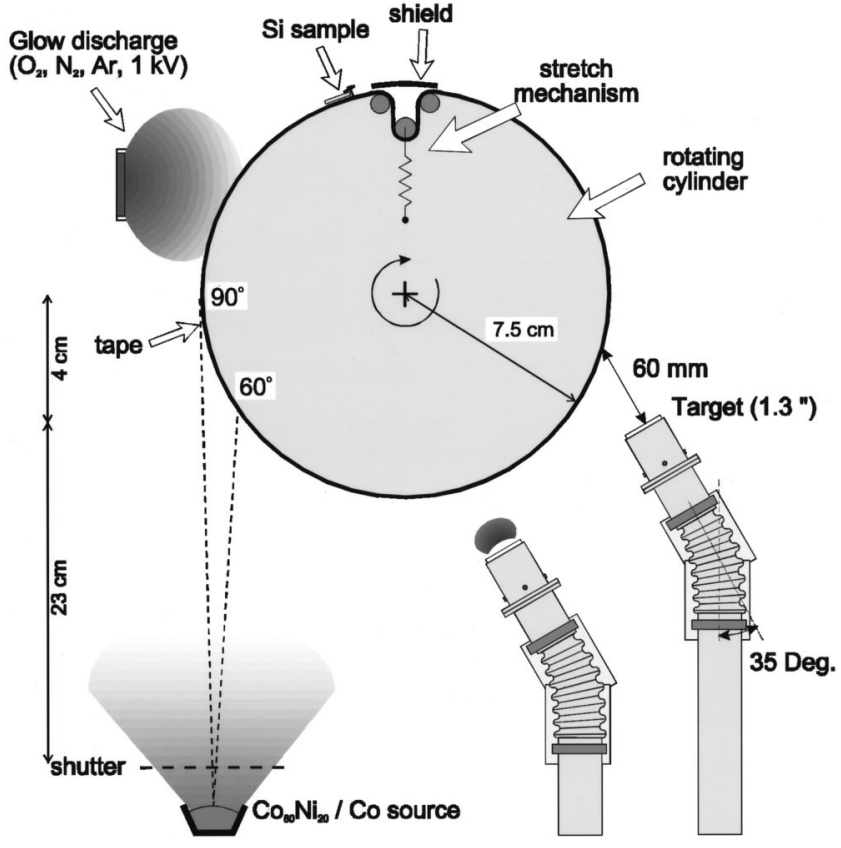

FIG. 1. Schematic view of the roll coater.

sputtering, evaporation can also be used in the same deposition system (bottom left of Fig. 1). Prior to deposition the substrates are cleaned by a glow discharge.

The sputtered sample that is discussed in this article was deposited from a pure cobalt target by dc magnetron sputtering. The optimum sputtering conditions were determined from previous experiments with a static trial setup, i.e., no rotation of the rotating cylinder, as was described in Ref. 13. No oxygen was added during the process, while an argon pressure of $2.5 \times 10^{-3}$ mbar and a sputter power of $50 \mathrm{~W}$ were used. As a substrate an endless PET film (Teijin Q68 S) with a thickness of $10 \mu \mathrm{m}$ was employed (see also Ref. 7).

\section{PREPARATION OF CROSS-SECTION TEM SAMPLES}

TEM cross sections of MS or ME tapes pose several unique problems during preparation. In this article we present a recipe developed at the Central Materials Analysis Laboratory of MESA+ [formerly the Central Laboratory of the Centre for Materials Research (CMO)] which is an adapted version of the conventional preparation of "standard" cross sections. If this recipe is followed closely one can make TEM cross sections of MS or ME tapes which are large homogeneous electron transparent, thin to ultrathin and leaving the very fragile polymer tape substrate and glue layer completely intact. A typical example is illustrated in this article. It shows a TEM image of a MS film with clear columnar structure on a polymer base film, in cross section. Precise control during all stages is essential and first of all, applying the appropriate glue to make the cross-section sandwich at room temperature, stopping the dimpling stage at the right moment (not too thin to avoid mechanical damage of the fragile plastic substrate), and finally, using argon ion
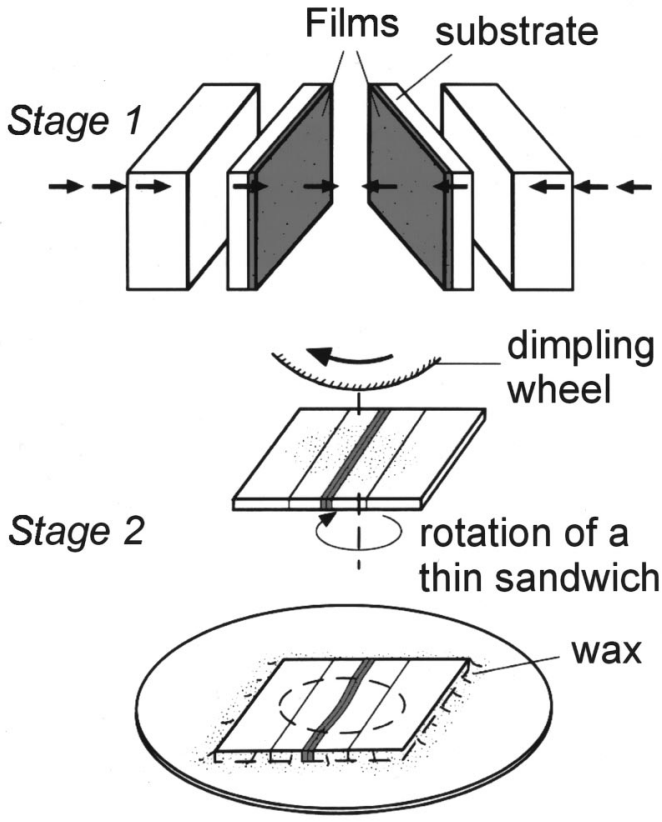

Stage 3

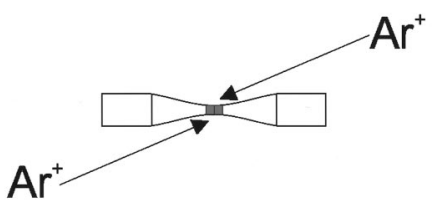

FIG. 2. Schematic representation of the various stages involved in making a TEM specimen in cross section. In Stage 1 the relevant parts including the buffer material are glued to each other with a two-component resin. After curing the sandwich is ground in such a fashion that a cylindrically shaped bar appears. That bar is then cut into thin slices, one slice is then ground down to a thickness of $200 \mu \mathrm{m}$. Stage 2 involves dimpling each side of the slice until for the central part of the cross section a thickness of 15-20 mm is reached. In Stage 3 the dimpled sandwich is finally made electron transparent via dual ion milling.

beams with an incidence angle as low as possible, and most important, terminating the ion thinning process just before a hole is created. It is essential in this case that the operator keeps his eye continuously on the ion thinning process.

Figure 2 is a schematic representation of the standard procedure for TEM specimen preparation in cross section. The preparation of a MS or ME tape in cross section is very similar to the procedure as depicted in Fig. 2, with a few significant differences. The most important aspect to bear in mind is that the use of heating steps should be limited as much as possible to avoid wrinkling and distortion of the plastic tape, in particular with epoxy resins that cure at elevated temperatures. That means that the application of GATAN G-1 epoxy + hardener or M-Bond610, which are normally used to glue all parts of the sandwich (stage 1 in Fig. 2), is not suitable since the optimum curing temperature ranges between 150 and $180^{\circ} \mathrm{C}$. Instead we applied a twocomponent resin that cures at room temperature, Araldit version $\mathrm{h} 0302$. We found out, however, that the optimal curing time is at least $48 \mathrm{~h}$, the sandwich components being held together under a light but steady pressure (we put a weight of $\sim 100 \mathrm{~g}$ on top of the sandwich pile). Following these rules we found that the hardened sandwich is at least as stable as 


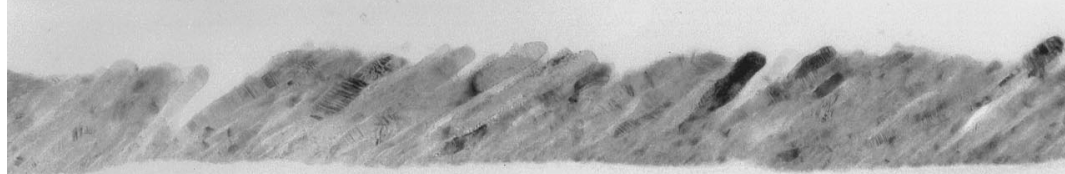

(a)

\section{$\overline{100 \mathrm{~nm}}$}

(b)

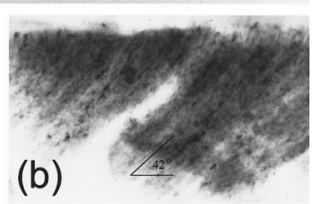

FIG. 3. (a) Low-magnification TEM image of the MS tape in cross section prepared according to the recipe described in this article. (b) Shows an image of a commercial Hi8 ME tape as prepared using our previous GATAN model 600 dual ion miller. Note that the experimental MS tape (a) has been obtained without rotating the drum during sputtering (see Fig. 1). The Hi8 ME tape (b), however, has been created with drum rotation. The scale bar in this figure represents both (a) and (b). one in which M-Bond610 or GATAN G-1 epoxy-resin is used.

For best results the following procedure should be used: take a very clean and dry microscope slide $(\sim 26 \times 76 \mathrm{~mm})$ and attach it firmly to a (flat) working table by applying some glue or the use of double-sided adhesive tape. Then put a piece of magnetic tape with its film side on the glass plate holding both ends of the magnetic tape with two pieces of Scotch transparent adhesive tape attached to the polymer side of the magnetic tape. If the magnetic tape is flat and stretched (no curls) attach the magnetic tape to the glass plate by pressing the two pieces of Scotch adhesive tape onto the glass plate. Next step is gluing two buffer substrates to the magnetic tape. For substrate material take silicon cut into slices of $3 \times 10 \mathrm{~mm}$. Now apply a minute amount of Araldit h0302 to each of the first two Si buffer slices, take care to create a smooth glue layer on the silicon surface, and attach them to the (polymer side of the magnetic tape). While attaching the move the slices carefully slightly forward and backward so that the glue layer is very evenly distributed between Si slice and magnetic tape. Glue additional Si slices on the back of each of the first slices until the total thickness of each stack is about $1.5 \mathrm{~mm}$ in height. You should now have two silicon stacks "sitting" next to each other and both being attached to the backside of the magnetic tape. In order to keep the stacks immobile during the curing period take a large piece of Scotch adhesive tape and attach both ends of that piece of strip to the left and right side of the glass plate covering both neighboring silicon stacks. If it looks satisfying and stable put a weight with a flat bottom on top of the two stacks. The weight should be $\sim 100 \mathrm{~g}$. Leave the spot undisturbed and let the stacks cure for at least $48 \mathrm{~h}$.

After the curing period, remove the Scotch adhesive tape and free the two stacks by carefully cutting the magnetic tape with a sharp knife around the stack border $(\sim 3 \times 10 \mathrm{~mm})$. The final step is the attachment of the two stacks. In order to do so carefully apply a minute amount of Araldit h0302 on the magnetic film surface of one of the stacks. Press the other stack with the magnetic film surface onto the first one so that a sandwich is created. Again let this sandwich cure for at least another $48 \mathrm{~h}$ with the same weight of $100 \mathrm{~g}$. Apply sufficient Scotch adhesive tape so that the sandwich is immobile during the second curing period.

After curing, the sandwich has to be shaped into a cylinder in such a fashion that the diameter is equal to $3.05 \mathrm{~mm}$.
For that purpose we use waterproof abrasive paper with 500 grit. From the cylindrically shaped sandwich we then cut out a slice with a thickness of $350 \mu \mathrm{m}$ which has to be ground further down to $190 \mu \mathrm{m}$ using again waterproof abrasive paper with 500 grit.

Mechanical thinning continues by dimpling this slice on both sides further down until the center part has reached a thickness of $\sim 15-20 \mu \mathrm{m}$ (stage 2 in Fig. 2). Reducing this thickness further down will result in a very fragile and/or damaged interface region in our experience! If this has occurred the specimen can be thrown away since ion sputtering will not "heal" the interface. At all mechanical thinning stages the wax (purchased from GATAN) used to hold the specimen on a suitable sample stub was applied/removed by heating the sample stub to approximately $70-90{ }^{\circ} \mathrm{C}$ as shortly as possible. Removal of residual wax on the specimen was achieved by rinsing it in acetone.

The final thinning stage; stage 3 in Fig. 2, is very critical. For that purpose we used the GATAN model 691 Precision Ion Polishing System (PIPS), with an incidence angle as low as $\pm 3^{\circ}$ for both ion guns (one for on-top sputtering, the other one hitting the dimpled slice from the bottom side) during $30-40 \mathrm{~min}$. It is essential in this case that the operator keeps his eye continuously on the ion thinning process. The very final stage is very important; as soon as a hole starts to appear the ion thinning process should be stopped immediately.

The TEM images were recorded in a Philips CM30 Twin/ STEM operated at $300 \mathrm{kV}$.

\section{RESULTS}

Figure 3(a) shows a low-magnification image of an experimental MS tape in cross section. From this image it can be seen that homogeneous electron transparency is achieved for several microns along the tape. Moreover, the underlying polymer substrate and glue layer are both intact. For comparison, we also show a TEM image [Fig. 3(b)] in cross section of a commercial Hi8 ME tape. The brittle metal layer in (a) is clearly mechanically less compromised as compared to the picture in (b). The particular example shown in the bottom picture was prepared using a previous recipe, one of the most relevant differences being the implementation of the GATAN-model 600-dual ion miller. This ion miller only allowed useful sputtering angles down to $\sim 12^{\circ}$. The GATAN 691 PIPS, on the other hand, can yield precisely fo- 
cused ion beams at near glancing incidence (from $10^{\circ}$ to $1^{\circ}$ ). Moreover, with the PIPS it is much more easier to detect the onset of an etch hole at which the etch process should be terminated promptly (see also Sec. III), resulting in a large homogenous transparent and mechanically stable tapered section. If, for example, the etch process is stopped even after a very small but clear etch hole is created, the glue layer in which the thin film has been embedded will, in our experience, not be stable enough to support this film, resulting in curling of the film.

\section{CONCLUSIONS}

In conclusion we have shown that our recipe for making cross-sectional TEM specimens is especially beneficial for brittle metal layers even on polymer base films. This recipe proves to be a good alternative to the use of a microtome.
${ }^{1}$ J. Jodge and P. Tang (private communication).

${ }^{2} \mathrm{H}$. te Lintelo (private communication).

${ }^{3}$ S. Honodera, H. Kondo, and T. Kawana, MRS Bull. 21, 35 (1996).

${ }^{4}$ H. Ho, G. Gau, and G. Thomas, J. Appl. Phys. 65, 3161 (1989).

${ }^{5}$ K. Sato, K. Chiba, T. Ito, T. Ssaki, and J. Hokkyo, J. Appl. Phys. 69, 4736 (1991).

${ }^{6}$ J. Hokkyo, T. Suzuki, K. Chiba, K. Sato, Y. Arisaka, T. Sasaki, and Y. Ebine, J. Magn. Magn. Mater. 120, 281 (1993).

${ }^{7}$ P. ten Berge, L. Abelmann, J. C. Lodder, A. Schrader, and S. Luitjens, J. Magn. Soc. Jpn. 18, 295 (1994).

${ }^{8}$ M. D. Bijker, E. M. Visser, and J. C. Lodder, Tribol. Int. 31, 553 (1998).

${ }^{9}$ H. J. Richter, IEEE Trans. Magn. 29, 21 (1993).

${ }^{10}$ J. P. C. Bernards and C. P. G. Schrauwen, Ph.D. thesis, University of Twente, 1990.

${ }^{11}$ Y. Maeda (private communication).

${ }^{12}$ K. Ozawa, H. Masuya, and M. Takahashi, J. Magn. Magn. Mater. 40, 175 (1983).

${ }^{13}$ M. D. Bijker, E. M. Visser, J. C. Lodder, and Th. J. A. Popma, J. Magn. Magn. Mater. 193, 352 (1999). 